\title{
Microbial profiling during anaerobic digestion of cheese whey in reactors operated at different conditions
}

Treu, Laura; Tsapekos, Panagiotis; Peprah, Maria; Campanaro, Stefano; Giacomini, Alessio; Corich, Viviana; Kougias, Panagiotis ; Angelidaki, Irini

\section{Published in:}

Bioresource Technology

Link to article, DOI:

10.1016/j.biortech.2018.12.084

Publication date:

2019

Document Version

Peer reviewed version

Link back to DTU Orbit

Citation (APA):

Treu, L., Tsapekos, P., Peprah, M., Campanaro, S., Giacomini, A., Corich, V., Kougias, P., \& Angelidaki, I. (2019). Microbial profiling during anaerobic digestion of cheese whey in reactors operated at different conditions. Bioresource Technology, 275, 375-385. https://doi.org/10.1016/j.biortech.2018.12.084

\section{General rights}

Copyright and moral rights for the publications made accessible in the public portal are retained by the authors and/or other copyright owners and it is a condition of accessing publications that users recognise and abide by the legal requirements associated with these rights.

- Users may download and print one copy of any publication from the public portal for the purpose of private study or research.

- You may not further distribute the material or use it for any profit-making activity or commercial gain

- You may freely distribute the URL identifying the publication in the public portal 


\title{
Microbial translocation revisited: targeting the endotoxic potential of gut microbes in $\mathrm{HIV}$-infected individuals
}

\author{
Short title: Endotoxic potential of the gut microbiome
}

Christopher STORM-LARSEN ${ }^{1,2,3}$, Birgitte STIKSRUD ${ }^{3,4}$, Carsten ERIKSEN ${ }^{5}$, Piotr NOWAK 7 , Kristian HOLM ${ }^{1,2,3}$, Anders THALME ${ }^{7}$, Anne Ma DYRHOL-RIISE ${ }^{3,4,6,8}$, Susanne BRIX ${ }^{5}$, Johannes $\mathrm{R} \mathrm{HOV}^{1,2,3,8,9 *}$, Marius TRØSEID ${ }^{2,4,8,10} *$

1 Norwegian PSC Research Center, Department of Transplantation Medicine,

Oslo University Hospital, Oslo, Norway

2 Research Institute of Internal Medicine, Division of Cancer Medicine, Surgery and Transplantation, Oslo University Hospital, Oslo, Norway

3 Institute of Clinical Medicine, University of Oslo, Oslo, Norway

4 Department of Infectious Diseases, Oslo University Hospital, Oslo, Norway

5 Department of Biotechnology and Biomedicine, Technical University of Denmark, Kgs Lyngby, Denmark

6 Department of Clinical Science, Faculty of Medicine, University of Bergen, N-5020 Bergen, Norway

7 Department of Infectious Diseases, Karolinska University Hospital, Stockholm, Sweden

8 K.G. Jebsen Center for Inflammation Research, Institute of Clinical Medicine, University of Oslo, Norway

9 Section of Gastroenterology, Department of Transplantation Medicine, Oslo University Hospital, Norway

10 Section of Clinical Immunology and Infectious Diseases, Oslo University Hospital, Oslo, Norway

*These authors share senior authorship

Corresponding authors: marius.troseid@medisin.uio.no and christopher@storm-larsen.no 
Objective: Translocation of microbial products such as lipopolysaccharides (LPS) from the gut may contribute to chronic inflammation in HIV-infected individuals. Recent studies indicate that differences in degree of acylation of gut-bacterial derived LPS may explain variable immune effects, with hexa-acylated rather than penta-acylated LPS having pro-inflammatory capacity. We investigated whether the degree of acylation of gut-derived LPS associates with systemic inflammation, and the potential effect of probiotic intervention.

Methods: Gut microbiota profiles from a probiotics intervention were investigated, and validated in a cohort of HIV-infected individuals commencing ART. The PiCRUSt software was used to predict overall functional capacity of the microbiota, and in-house bioinformatics to distinguish between bacteria producing hexa- and penta-acylated LPS.

Results and conclusion: HIV-infected individuals with the highest ratio of pro-inflammatory hexaacylated LPS- to non-inflammatory penta-acylated LPS-producing bacteria exhibited increased levels of systemic inflammation (neopterin, $\mathrm{P}<0.001$ ) and tryptophan catabolism (kynurenine/tryptophanratio, $\mathrm{P}=0.01$ ), indicating a link between pro-inflammatory LPS, tryptophan catabolism and inflammation. After probiotics for eight weeks, there was a decrease in gram-negative bacteria $(\mathrm{P}=0.01)$, related primarily to a reduction in bacteria producing penta-acylated LPS $(\mathrm{P}=0.01)$, but not hexa-acylated LPS. The reduction in gram-negative bacteria correlated positively with decreased plasma LPS $(\mathrm{r}=0.72)$, mainly related to a reduction in bacteria producing non-inflammatory pentaacylated LPS ( $\mathrm{r}=0.58)$. Notably, gut bacteria producing hexa-acylated LPS were outnumbered by penta-acylated LPS with a factor of 25 in HIV-infected individuals. Further studies are warranted to determine whether microbes producing hexa-acylated LPS might be a more relevant trigger of systemic inflammation compared to plasma LPS captured by the existing limulus assay.

Keywords: HIV, probiotics, endotoxins, LPS, gastrointestinal microbiome, inflammation, limulus test 


\section{Background}

The widespread access to antiretroviral therapy (ART) for HIV-1 patients has changed the disease from a lethal to a chronic condition. However, the chronically infected patients exhibit an increased risk of non-AIDS morbidities such as cardiovascular, hepatic, renal or neurologic diseases. $(1,2)$ This phenomenon has been linked to the persistent low-grade inflammation observed in patients successfully treated with ART.(3-5)

The initial HIV infection causes a massive loss of Th17 positive CD4+ T-cells in the gutassociated lymphoid tissue (GALT). The depletion of these cells is associated with inflammation in the mucosa, apoptosis of epithelial cells and breakdown of tight junctions. $(6,7)$ It has been suggested that the loss of integrity of the gut and blood barrier may allow gut microbial products, such as lipopolysaccharide (LPS), to translocate into the portal and systemic circulation. LPS, a component of the outer wall of gram-negative bacteria, is a potent immune activator via interaction with the Tolllike receptor (TLR)-4 complex through recognition by MD-2 and CD14 co-receptors. $(8,9)$

In HIV-infected individuals, plasma LPS is elevated and potentially linked to systemic inflammation, activated $\mathrm{T}$ cells and cardiovascular risk factors.(6, 10-12) Furthermore, activated T cells produce interferon (IFN)- $\gamma$, which together with other cytokines activates tryptophan catabolism through upregulation of indolamine 2,3-dioxygenase (IDO-1).(7, 13) Moreover, microbial translocation and gut microbiota alterations associate with increased kynurenine to tryptophan ratio (KT-ratio), a measure of tryptophan degradation which is linked to systemic inflammation, disease progression and increased mortality in HIV-infected individuals.(7, 14, 15) The main pathway of tryptophan catabolism is to kynurenine, which has immunosuppressive properties, in part by inhibiting T-cell proliferation and depleting Th17 cells, which in turn weakens the mucosal barrier and promotes low grade endotoxemia, inflammation and $\mathrm{T}$ cell activation.(7) Activated $\mathrm{T}$ cells produce IFN- $\gamma$, which induce tryptophan catabolism through upregulation of IDO-1, and contributes to neopterin release by activated monocytes/macrophages. (13) (13)(13) Recent studies have explored the metagenomic heterogeneity of LPS and its capacity to activate a downstream immune response 
after binding to the TLR4 receptor complex.(16-20) The endotoxic activity of LPS depends on the degree of acylation of the lipid A moiety of the molecule. Some gram-negative bacteria produce a penta-acylated form, which antagonizes activation of human TLR4, whereas others produce a TLR4 activating hexa-acylated form of LPS.(21) Currently available biochemical assays capture all types of LPS independent of their pro-inflammatory effects, and to our knowledge no biochemical assay exists to determine the levels of the two LPS variants in blood. Revealing the relative abundances of, and ratio between bacteria producing penta-acylated and hexa-acylated LPS within the gut microbiota would therefore be a measure of the endotoxic pro-inflammatory potential of LPS, and has been proposed as a driver for asthma and type 1 diabetes. $(20,22)$

Several strategies have been applied to reduce systemic inflammation in HIV by targeting microbial translocation, including LPS-binding agents and antibiotics, but with mostly negative results. $(23,24)$ In a previous study we reported a reduction in levels of D-dimer, and a tendency to reduced levels of CRP and IL-6 after probiotic intervention in HIV-infected patients on stable ART.(25) However, no changes in markers of microbial translocation were observed.

In the present study we aimed to expand the analyses of microbial translocation in the same patient cohort by focusing on the gut metagenome, specifically addressing the biosynthesis of LPS and the ratio of hexa:penta-acylated LPS-producing bacteria. We hypothesized that i) alterations of gut microbial genes related to LPS biosynthesis and degree of acylation are related to markers of inflammation and tryptophan degradation in HIV-infected patients, ii) the effects of probiotic intervention in HIV-infected are associated with alterations of gut microbial genes related to LPS biosynthesis, in particular genes related to hexa-acylated LPS.

\section{Methods}

\section{Study participants}

We investigated a subgroup with available gut microbiota profiles $(\mathrm{n}=22)$ from a previously published probiotics intervention. For a complete methodology and baseline characteristics, we refer to supplementary table 1, http://links.Iww.com/QAD/B402 and the initial study.(25) In brief, HIV- 
infected $>18$ years old with HIV-RNA $<50$ copies per milliliter for at least 6 months and CD4 count $<500$ cells per microliter and with available $16 \mathrm{~S}$ ribosomal RNA (rRNA)-based gut microbiota profiles were included ( $n=10$ in the probiotic and $n=12$ in the control group). In the intervention group, the patients received $250 \mathrm{~mL}$ fermented skimmed milk supplemented with Lactobacillus rhamnosus $\left(10^{8} \mathrm{cfu} / \mathrm{mL}\right)$, Bifidobacterium animalis subsp. Lactis $\left(10^{8} \mathrm{cfu} / \mathrm{mL}\right)$ and Lactobacillus acidophilus La-5 $\left(10^{7} \mathrm{cfu} / \mathrm{mL}\right)$ for eight weeks, as previously described. In addition, we included a validation cohort consisting of HIV-infected individuals commencing ART, including available microbiota samples from HIV-infected individuals sampled 10 (4-15) months after introduction of ART ( $\mathrm{n}=16)$, non-infected controls (household members/partners of HIV-infected individuals, consisting mainly of MSM, n=9) and three HIV-infected elite controllers, which was sampled and sequenced using the same pipeline as in the probiotics intervention.(26)

\section{Soluble factors}

As previously described, total plasma LPS was analyzed by limulus amebocyte lysate colorimetric assay (Lonza, Walkersville, MD).(27) Soluble CD14 (sCD14) was analyzed by Quantikine ELISA kits (R\&D Systems Europe, Abingdon, United Kingdom).(25) Intestinal fatty acid binding protein (IFABP) was analyzed by ELISA kit (Hycult biotech, Uden, The Netherlands). Extracted DNA was sequenced on the Illumina MiSeq platform, targeting the V3-V4 region of the 16s rRNA. Reads were subsampled/rarefied to 9442 reads per sample and mapped using default values in "closed reference operational taxonomic unit (OTU) clustering" in QIIME 1.8.0 against the Greengenes database version 1308.(28)

\section{Microbial genes analysis}

Two different methods were used to determine microbial genes related to biosynthesis of LPS. The software PICRUSt was used to predict the genetic content of the metagenome based on the 16s rRNA sequencing data, which was subsequently assigned to KEGG (Kyoto Encyclopedia of Genes and Genome) pathways.(29) Available genera from the 16s rRNA sequencing were classified as gramnegative, and further separated into hexa-acylated, penta-acylated or tetra-acylated lipid A producing 
bacteria, or as non-LPS producing bacteria involving all gram-positive species, with the exception of Veillonella. Classification of bacteria into hexa-, penta- or tetra-acylated LPS producers was based on genomic information from all whole genome-sequenced deposited at the nucleotide database NCBI (www.ncbi.nlm.nih.gov), and then coupled to the taxonomic identification based on 16s rRNA gene sequences. Bacteria carrying the $L p x L$ gene, along with the remaining lipid A biosynthesis enzymes, produce the penta-acylated LPS variant, while carriers of both the $L p x L$ and $L p x M$ genes generally produce the proinflammatory hexa-acylated LPS. Bacteria carrying lipid A biosynthesis enzymes but neither $L p x L$ or $L p x M$ were classified as a tetra-acylated LPS producer.

\section{Statistical analysis}

All continuous variables are presented as median and interquartile range (IQR). Nonparametric statistics were applied, using Wilcoxon matched pairs test, Mann-Whitney U test and Spearman's rho test, as appropriate. Statistical correction for multiple testing were not applied because of the low sample size and the exploratory focus of the study. A two-tailed significance level of 0.05 was used. Statistical analyses were performed in SPSS Statistics v24.0 (IBM Corporation, Armonk, NY, USA). Graphical presentations were made using Prism V7.0d software (GraphPad, San Diego, CA).

\section{Results}

Penta-acylated bacteria outnumber hexa-acylated bacteria in HIV-infected individuals

In the total study population at baseline in the probiotic intervention cohort $(n=22)$, the median of the ratio of hexa:penta-acylated LPS-producing bacteria was 0.04 (0.01-0.11, fig. 1A), hence pentaacylated bacteria outnumbered hexa-acylated bacteria with a factor of 25 in HIV-infected individuals.

To further evaluate the magnitude of this ratio, we externally validated our results in a previously published cohort of HIV-infected individuals commencing ART, finding the same fraction of bacteria producing hexa and penta-acylated LPS (median ratio $0.03(0.01-0.21), \mathrm{P}=0.69)$. This validation cohort also consisted of non-infected controls $(n=9)$ and three HIV-infected elite 
controllers. Although the hexa:penta-ratio was not significantly lower in non-infected controls $(0.02$ (0.01-0.10), $\mathrm{P}=0.39)$, the elite controllers had substantially lower levels of hexa:penta-ratio compared to HIV-infected individuals on ART (median 0.001, $\mathrm{P}=0.002$, Fig.1A).

As some microbes can express several degrees of LPS-acylation, we also aimed to examine tetra-acylated LPS. However, no microbes (as defined by the genus level resolution available from 16s rRNA sequencing) expressing tetra-acylated LPS were found in the gut microbiota in any of the included patients in the present study.

Ratio of hexa:penta-acylated LPS-producing bacteria is related to neopterin and tryptophan catabolism in HIV-infected individuals

Since increased relative levels of pro-inflammatory hexa-acylated LPS-producing species may be an indicator of enhanced gut inflammatory status, we next explored whether the ratio of hexa:pentaacylated LPS-producing bacteria in the gut (hexa:penta-ratio), at baseline in the probiotic intervention cohort, could be related to markers of systemic inflammation. We identified a significant correlation between plasma neopterin and the hexa:penta-ratio $(\mathrm{r}=0.59, \mathrm{P}=0.01$, fig. $1 \mathrm{~B})$, suggesting that a more pro-inflammatory milieu within the gram-negative bacteria in the gut is linked with systemic neopterin, which is released from activated monocytes mainly after IFN- $\gamma$ stimulation.(13)

Furthermore, when dichotomizing the dataset according to the median ratio of hexa:pentaratio, the group with the highest ratio not only had significantly higher levels of neopterin $(\mathrm{P}<0.001$, fig. 1C), but also an increased KT-ratio as a measure of tryptophan catabolism ( $\mathrm{P}=0.01$, fig. 1D). Furthermore, plasma levels of neopterin were strongly correlated with KT-ratio $(r=0.67, \mathrm{P}=0.001)$. No significant differences between the high and low hexa:penta-ratio groups were observed for CRP, Ddimer, IL-6, IFABP, total plasma LPS or sCD14 (Supplementary table 2 and 3, http://links.Iww.com/QAD/B402), or any of the HIV-related factors detailed in Supplementary table 
1, http://links.Iww.com/QAD/B402 (MSM vs non-MSM, current CD4 count, nadir CD4 count, duration of ART).

We did not find any positive correlation between KT-ratio and hexa:penta-ratio in the noninfected controls $(n=9)$, in fact there was a non-significant negative correlation $(r=-0.40, P=0.29)$ and a tendency to lower KT-ratio in individuals with the highest hexa:penta-ratio $(\mathrm{P}=0.19$, Fig. $1 \mathrm{E})$ in the control group.

Probiotic intervention in HIV-infected individuals is associated with decrease in overall LPS biosynthesis related primarily to non-inflammatory penta-acylated LPS-producing gram-negative bacteria

We next explored the effect of probiotics on LPS biosynthesis, and in line with the original study of the complete set of participants (25), no changes were observed after the intervention in relation to plasma LPS levels in the subgroup with available microbiota profiles $(\mathrm{P}=0.65$, table 1$)$. Furthermore, we performed genome-based bioinformatics analysis of the LPS-producing capacity within the gut microbiota, finding a significant reduction after probiotics intervention in median gene counts related to the KEGG pathway involved in overall LPS biosynthesis: 13894 (5256-19683) to 9683 (482714200) $(-30 \%, \mathrm{P}=0.01$, fig. $2 \mathrm{~A})$. This reduction was paralleled by a change in relative abundance of gram-negative bacteria (decreased from $0.27(0.11-0.55)$ to $0.12(0.05-0.18), \mathrm{P}=0.01$, table 2$)$, and a strong correlation between the two ( $\mathrm{r}=0.88, \mathrm{P}=0.001$, fig. $2 \mathrm{~B})$.

The decrease in gram-negative bacteria was mirrored by a reduction in the relative abundance of gram-negative bacteria with capacity for penta-acylated LPS production (from a median of 0.38 $(0.11-0.59)$ to $0.18(0.07-0.27)(\mathrm{P}=0.01$, fig. $2 \mathrm{C}$, table 2$))$, while no significant changes were seen for bacteria producing the pro-inflammatory hexa-acylated LPS (Fig. 2D, table 2). Notably, the abundances of hexa-acylated LPS-producing gut bacteria were generally very low (from 0.01 (0.002$0.01)$ to $0.002(<0.001-0.02)$, Fig. 2D). Likewise, no significant changes were seen in the ratio of hexa:penta-acylated LPS-producing bacteria $(0.01(0.01-0.04)$ to $0.03(0.01-0.08), \mathrm{P}=0.29)$ after probiotics administration (Fig. 2E). 
When focusing on which penta-acylated LPS-producing bacteria were reduced, we found a statistically significant reduction of Bacteroides (from relative abundance of $0.19(0.04-0.39)$ to 0.03 (0.002-0.09), $\mathrm{P}=0.01$, table 2 ), to be counter-regulated by increased abundances of total grampositives after the probiotics intervention (Table 2). In this regard we identified a negative correlation between the decrease of gram-negative bacteria and the increase of the supplemented Bifidobacterium $(\mathrm{r}=-0.72, \mathrm{P}=0.02)$ and Lactobacillus $(\mathrm{r}=-0.68, \mathrm{P}=0.03)$. No changes were observed in the control group (Table 2).

Reduction in gut bacteria producing penta-acylated LPS during probiotic intervention correlate with changes in plasma levels of LPS

When focusing on changes in the relative abundance of gram-negative bacteria in relation to systemic markers of microbial translocation and inflammation, we found that the decrease in gram-negative bacteria correlated positively with decreased plasma LPS ( $\mathrm{r}=0.72, \mathrm{P}=0.02$, fig. 3A) during the intervention, but not with changes in levels of CRP, IL-6, sCD14, D-dimer, neopterin or KT-ratio (Supplementary table 4, http://links.Iww.com/QAD/B402). Of note, the reduction in relative abundance of bacteria producing penta-acylated LPS showed the same trend for correlation with changes in total plasma LPS ( $\mathrm{r}=0.58, \mathrm{P}=0.08$, fig. $3 \mathrm{~B}$ ), whereas no such correlation was seen for bacteria producing hexa-acylated LPS (Supplementary table 4, http://links.Iww.com/QAD/B402).

\section{Discussion}

In the present study we focused on the biosynthesis and acylation degree of LPS by the gut microbiome, aiming to investigate whether the degree of acylation of gut-derived LPS associates with markers of systemic inflammation, and the potential effect of probiotic intervention in HIV-infected individuals. The main findings can be summarized as follows: 1. A higher ratio of bacteria producing hexa:penta-acylated LPS at baseline in the probiotic intervention cohort was associated with elevated markers of tryptophan degradation and the pro-inflammatory marker neopterin, reflecting an 
association between the LPS type ratio in the gut and the systemic inflammatory status. 2. A reduction in gut microbial gene counts related to biosynthesis of LPS was observed after probiotic intervention, mainly driven by a reduction in bacteria producing penta-acylated, but not hexa-acylated LPS. 3 . Changes in penta-acylated but not hexa-acylated LPS correlated closely with changes in plasma LPS, suggesting that LPS levels captured by the limulus assay to a large degree reflect non-inflammatory LPS.

It should be noted that hexa-acylated LPS producing bacteria had a very low abundance with a ratio of 1 to 25 as compared to penta-acylated LPS in HIV-infected individuals. To our knowledge, no such data are available from other HIV cohorts, and future studies should assess the magnitude of this ratio in a healthy background population. Possibly, low frequency microbes including bacteria producing hexa-acylated LPS might be relevant for systemic inflammation and metabolism, as suggested by the association between ratio of bacteria producing hexa:penta-acylated LPS and tryptophan catabolism, as well as to plasma neopterin.

Taken together, our observations could indicate that the ratio of bacterial genes related to hexa:penta-acylated LPS is a relevant measure of the microbial stimulatory capacity related to systemic inflammation in chronic HIV-infection. However, this ratio is a simplified measure of the overall interplay between microbes expressing different forms of LPS and the immune system, and acylation of LPS may not be the sole determinant of TLR4-responsiveness. In fact, some microbes such as Porhyromonas can produce and exploit multiple acylated forms of LPS, including tetra-, penta- and hexa-acylation to evade the innate immune system(30). However, in the present study cohorts, we could not identify any microbes expressing tetra-acylated LPS. It should also be noted that some strains of Bacteroides have the capacity to produce penta-acylated LPS with highly divergent potency(31). These variations are not captured by our bioinformatic approach taking advantage of $16 \mathrm{~s}$ data. Hence, direct quantification and functional analyses of differentially acylated forms of LPS would add valuable information in future studies. 
In several studies of the gut microbiome in HIV-infected individuals, significant differences are mainly reported among highly abundant bacterial taxa, such as increased abundance of Prevotella and reduced abundance of Bacteroides.(32) Recently, MSM-status was identified as an important confounder of the HIV-associated Prevotella-rich enterotype as opposed to higher abundance of Bacteroides in non-MSM, irrespective of HIV status.(33) Of note, Prevotella and Bacteroides both produce penta-acylated LPS, and apparently, hexa:penta-ratio was not associated with MSM status in the present study. In the non-infected individuals from the validation cohort, consisting of household members/partners of HIV-infected individuals, mostly MSM, we did not find significantly lower ratio of bacterial genes related to hexa:penta-acylated LPS, although this ratio was much lower in three elite controllers. However, the study cohorts are limited by small sample size, and we acknowledge that our findings, although intriguing, need to be replicated in larger, properly designed cohorts of HIV-infected individuals of various stages of immune status (including elite controllers and immunological non-responders) and appropriate controls (non-infected MSM and non MSM). Another limitation is that the genetic microbial potential of LPS biosynthesis genes may not necessarily translate into circulating levels of hexa- and penta-acylated LPS, which could be affected by probiotic intervention. Although no assay currently exists that can distinguish inhibitory from inflammatory LPS in the periphery(34), there is clearly a need for such a kit in future work of this emerging field of research.

Probiotic intervention did not alter the abundance of hexa-acylated LPS-producing bacteria, corresponding with no effect on plasma levels of neopterin, KT-ratio or sCD14. Previous studies aiming to reduce microbial translocation have not shown effects on systemic total LPS levels in HIVinfected individuals. $(23,24)$ Rifaximin, a non-absorbed oral antibiotic, decreased total plasma levels of LPS by $50 \%$ in patients with alcoholic cirrhosis, however no effects were seen when rifaximin was given to ART-treated HIV patients. $(24,35)$ Sevelamer carbonate, a phosphate-lowering drug, decreases circulating LPS levels in patients with renal insufficiency. $(36,37)$ Yet, no such effects were seen when administered to HIV-infected individuals.(23) Our results indicate that although we found a reduction in the total abundance of LPS-producing gut bacteria, this reduction was mainly explained 
by a decrease in the genus of Bacteroides, which produces penta-acylated LPS. Of note, no changes were seen in the ratio of hexa:penta-acylated LPS-producing bacteria after probiotic intervention, suggesting no effect on the overall endotoxic potential of the gut microbiome.

A large number of studies focusing on microbial translocation in HIV have been published since Brenchley et al. first described the phenomenon in 2006.(6) To our knowledge, none of these studies have investigated differences in the endotoxic potential of LPS. Importantly, measuring LPS in plasma would be a biomarker of microbial translocation, but in order to study LPS as an immune activator, there is a need to distinguish between the different forms and the inflammatory potential of this toxin. In our data, the ratio between genes related to hexa-acylated, pro-inflammatory LPS, and inhibitory penta-acylated LPS in the gut microbiota varied greatly between individuals; with numbers ranging from $1 / 500$ to $1 / 10$. In addition, the close correlation between reduction in microbes producing penta-acylated LPS and changes in systemic total LPS levels, suggest that measurements of total plasma LPS reflect mainly penta-acylated LPS, which has a much lower TLR4 stimulatory potential than hexa-acylated LPS. This point is of relevance since the most frequently used limulus assay (Lonza) used for measurement of plasma LPS cannot distinguish between LPS variants with different degrees of acylation.(38) This could explain the low to moderate correlations between total plasma LPS levels and inflammatory markers in the literature $(6,39,40)$; which was also replicated in this study. In light of our findings, one could question whether plasma LPS measured by the limulus assay is a relevant biomarker in trials targeting the gut microbiome and/or microbial translocation.

In conclusion, we identified an association between increased ratio of hexa:penta-acylated LPS-producing bacteria and systemic inflammation measured as neopterin and tryptophan catabolism in HIV-infected individuals. The reduction in penta-acylated but not pro-inflammatory hexa-acylated LPS after probiotic intervention correlated closely with changes in plasma LPS suggesting that LPS levels captured by the limulus assay to a large degree reflects non-inflammatory LPS. Notably, gut bacteria producing hexa-acylated LPS varied substantially between individuals, and were vastly outnumbered by penta-acylated LPS. Further studies are warranted to determine whether the ratio of hexa:penta-acylated LPS-producing bacteria might be a more relevant measure for trigger of systemic 
inflammation than the currently existing limulus assay, and whether the hexa:penta-ratio could relate to disease progression and clinical outcome in HIV-infected individuals.

\section{Acknowledgements}

We thank medical technologist Jeanette Steen, Center for Clinical Heart Research, Oslo University Hospital Ullevål, for analysis of I-FABP. 


\section{References:}

1. Deeks SG, Tracy R, Douek DC. Systemic effects of inflammation on health during chronic HIV infection. Immunity. 2013;39(4):633-45.

2. Freiberg MS, Chang CC, Kuller LH, Skanderson M, Lowy E, Kraemer KL, et al. HIV infection and the risk of acute myocardial infarction. JAMA Intern Med. 2013;173(8):614-22.

3. Burdo TH, Lo J, Abbara S, Wei J, DeLelys ME, Preffer F, et al. Soluble CD163, a novel marker of activated macrophages, is elevated and associated with noncalcified coronary plaque in HIV-infected patients. J Infect Dis. 2011;204(8):1227-36.

4. Neuhaus J, Jacobs DR, Jr., Baker JV, Calmy A, Duprez D, La Rosa A, et al. Markers of inflammation, coagulation, and renal function are elevated in adults with HIV infection. J Infect Dis. 2010;201(12):1788-95.

5. Kuller LH, Tracy R, Belloso W, De Wit S, Drummond F, Lane HC, et al. Inflammatory and coagulation biomarkers and mortality in patients with HIV infection. PLoS Med. 2008;5(10):e203.

6. Brenchley JM, Price DA, Schacker TW, Asher TE, Silvestri G, Rao S, et al. Microbial translocation is a cause of systemic immune activation in chronic HIV infection. Nature medicine. 2006;12(12):1365-71.

7. Sandler NG, Douek DC. Microbial translocation in HIV infection: causes, consequences and treatment opportunities. Nat Rev Microbiol. 2012;10(9):655-66.

8. Gioannini TL, Weiss JP. Regulation of interactions of Gram-negative bacterial endotoxins with mammalian cells. Immunol Res. 2007;39(1-3):249-60.

9. Miller SI, Ernst RK, Bader MW. LPS, TLR4 and infectious disease diversity. Nat Rev Microbiol. 2005;3(1):36-46.

10. Nowroozalizadeh S, Mansson F, da Silva Z, Repits J, Dabo B, Pereira C, et al. Microbial translocation correlates with the severity of both HIV-1 and HIV-2 infections. J Infect Dis. 2010;201(8):1150-4.

11. Troseid M, Manner IW, Pedersen KK, Haissman JM, Kvale D, Nielsen SD. Microbial translocation and cardiometabolic risk factors in HIV infection. AIDS research and human retroviruses. 2014;30(6):514-22.

12. Pedersen KK, Pedersen M, Troseid M, Gaardbo JC, Lund TT, Thomsen C, et al. Microbial translocation in HIV infection is associated with dyslipidemia, insulin resistance, and risk of myocardial infarction. J Acquir Immune Defic Syndr. 2013;64(5):425-33.

13. Huber C, Batchelor JR, Fuchs D, Hausen A, Lang A, Niederwieser D, et al. Immune response-associated production of neopterin. Release from macrophages primarily under control of interferon-gamma. J Exp Med. 1984;160(1):310-6.

14. Vujkovic-Cvijin I, Dunham RM, Iwai S, Maher MC, Albright RG, Broadhurst MJ, et al. Dysbiosis of the gut microbiota is associated with HIV disease progression and tryptophan catabolism. Sci Transl Med. 2013;5(193):193ra91.

15. Hunt PW, Sinclair E, Rodriguez B, Shive C, Clagett B, Funderburg N, et al. Gut epithelial barrier dysfunction and innate immune activation predict mortality in treated HIV infection. J Infect Dis. 2014;210(8):1228-38. 
16. Gnauck A, Lentle RG, Kruger MC. The characteristics and function of bacterial lipopolysaccharides and their endotoxic potential in humans. International reviews of immunology. 2015:1-31.

17. Maeshima N, Fernandez R. Recognition of lipid A variants by the TLR4-MD-2 receptor complex. Frontiers in Cellular and Infection Microbiology. 2013;3.

18. Martirosyan A, Ohne Y, Degos C, Gorvel L, Moriyon I, Oh S, et al. Lipopolysaccharides with acylation defects potentiate TLR4 signaling and shape T cell responses. PLoS One. 2013;8(2):e55117.

19. Steimle A, Autenrieth IB, Frick JS. Structure and function: Lipid A modifications in commensals and pathogens. International journal of medical microbiology : IJMM. 2016.

20. Brix S, Eriksen C, Larsen JM, Bisgaard H. Metagenomic heterogeneity explains dual immune effects of endotoxins. The Journal of allergy and clinical immunology. 2015;135(1):277-80.

21. Rietschel ET, Kirikae T, Schade FU, Mamat U, Schmidt G, Loppnow H, et al. Bacterial endotoxin: molecular relationships of structure to activity and function. FASEB J. 1994;8(2):217-25.

22. Vatanen T, Kostic AD, d'Hennezel E, Siljander H, Franzosa EA, Yassour M, et al. Variation in Microbiome LPS Immunogenicity Contributes to Autoimmunity in Humans. Cell. 2016.

23. Sandler NG, Zhang X, Bosch RJ, Funderburg NT, Choi AI, Robinson JK, et al. Sevelamer does not decrease lipopolysaccharide or soluble CD14 levels but decreases soluble tissue factor, lowdensity lipoprotein (LDL) cholesterol, and oxidized LDL cholesterol levels in individuals with untreated HIV infection. J Infect Dis. 2014;210(10):1549-54.

24. Tenorio AR, Chan ES, Bosch RJ, Macatangay BJ, Read SW, Yesmin S, et al. Rifaximin has a marginal impact on microbial translocation, T-cell activation and inflammation in HIV-positive immune non-responders to antiretroviral therapy - ACTG A5286. J Infect Dis. 2015;211(5):780-90.

25. Stiksrud B, Nowak P, Nwosu FC, Kvale D, Thalme A, Sonnerborg A, et al. Reduced Levels of D-dimer and Changes in Gut Microbiota Composition After Probiotic Intervention in HIV-Infected Individuals on Stable ART. J Acquir Immune Defic Syndr. 2015;70(4):329-37.

26. Nowak P, Troseid M, Avershina E, Barqasho B, Neogi U, Holm K, et al. Gut microbiota diversity predicts immune status in HIV-1 infection. AIDS. 2015;29(18):2409-18.

27. Troseid M, Nowak P, Nystrom J, Lindkvist A, Abdurahman S, Sonnerborg A. Elevated plasma levels of lipopolysaccharide and high mobility group box-1 protein are associated with high viral load in HIV-1 infection: reduction by 2-year antiretroviral therapy. Aids. 2010;24(11):1733-7.

28. Caporaso JG, Kuczynski J, Stombaugh J, Bittinger K, Bushman FD, Costello EK, et al. QIIME allows analysis of high-throughput community sequencing data. Nat Methods. 2010;7(5):3356.

29. Langille MG, Zaneveld J, Caporaso JG, McDonald D, Knights D, Reyes JA, et al. Predictive functional profiling of microbial communities using $16 \mathrm{~S}$ rRNA marker gene sequences. Nat Biotechnol. 2013;31(9):814-21.

30. Ding PH, Wang CY, Darveau RP, Jin L. Porphyromonas gingivalis LPS stimulates the expression of LPS-binding protein in human oral keratinocytes in vitro. Innate Immun. 2013;19(1):66-75. 
31. Berezow AB, Ernst RK, Coats SR, Braham PH, Karimi-Naser LM, Darveau RP. The structurally similar, penta-acylated lipopolysaccharides of Porphyromonas gingivalis and Bacteroides elicit strikingly different innate immune responses. Microb Pathog. 2009;47(2):68-77.

32. Lozupone CA, Rhodes ME, Neff CP, Fontenot AP, Campbell TB, Palmer BE. HIV-induced alteration in gut microbiota: driving factors, consequences, and effects of antiretroviral therapy. Gut Microbes. 2014;5(4):562-70.

33. Noguera-Julian M, Rocafort M, Guillen Y, Rivera J, Casadella M, Nowak P, et al. Gut Microbiota Linked to Sexual Preference and HIV Infection. EBioMedicine. 2016;5:135-46.

34. d'Hennezel E, Abubucker S, Murphy LO, Cullen TW. Total Lipopolysaccharide from the Human Gut Microbiome Silences Toll-Like Receptor Signaling. mSystems. 2017;2(6).

35. Vlachogiannakos J, Saveriadis AS, Viazis N, Theodoropoulos I, Foudoulis K, Manolakopoulos S, et al. Intestinal decontamination improves liver haemodynamics in patients with alcohol-related decompensated cirrhosis. Alimentary pharmacology \& therapeutics. 2009;29(9):9929.

36. Stinghen AE, Goncalves SM, Bucharles S, Branco FS, Gruber B, Hauser AB, et al. Sevelamer decreases systemic inflammation in parallel to a reduction in endotoxemia. Blood purification. 2010;29(4):352-6.

37. Navarro-Gonzalez JF, Mora-Fernandez C, Muros de Fuentes M, Donate-Correa J, CazanaPerez V, Garcia-Perez J. Effect of phosphate binders on serum inflammatory profile, soluble CD14, and endotoxin levels in hemodialysis patients. Clinical journal of the American Society of Nephrology : CJASN. 2011;6(9):2272-9.

38. Gutsmann T, Howe J, Zahringer U, Garidel P, Schromm AB, Koch MH, et al. Structural prerequisites for endotoxic activity in the Limulus test as compared to cytokine production in mononuclear cells. Innate Immun. 2010;16(1):39-47.

39. Sandler NG, Wand H, Roque A, Law M, Nason MC, Nixon DE, et al. Plasma levels of soluble CD14 independently predict mortality in HIV infection. J Infect Dis. 2011;203(6):780-90.

40. Troseid M, Lind A, Nowak P, Barqasho B, Heger B, Lygren I, et al. Circulating levels of HMGB1 are correlated strongly with MD2 in HIV-infection: possible implication for TLR4-signalling and chronic immune activation. Innate Immun. 2013;19(3):290-7. 


\section{Figures legends}

\section{Figure 1. Ratio of hexa:penta-acylated LPS producers in the gut is related to systemic}

\section{inflammatory markers in HIV-infected individuals.}

A The ratio of gut bacteria producing hexa:penta-acylated LPS at baseline before probiotic intervention ( $\mathrm{n}=22$, black), HIV+ from a validation cohort after commencing $\operatorname{ART}(\mathrm{n}=16$, red), noninfected household-members of HIV+ $(n=9$, green $)$ and elite controllers $(n=3$, blue). B Hexa:pentaacylated LPS producers correlates with plasma levels of neopterin in HIV-infected. When dichotomizing, the patients with the highest ratio of gut bacteria producing hexa:penta-acylated LPS showed elevated levels of neopterin (C) and the KT-ratio (D). E In healthy controls there was a trend of lower levels of KT-ratio with higher hexa:penta-ratio.

KT-ratio: kynurenine-tryptophan-ratio, a measure of tryptophan degradation.

Mann-Whitney U test (a, c-e) and Spearman's rho test (b).

A

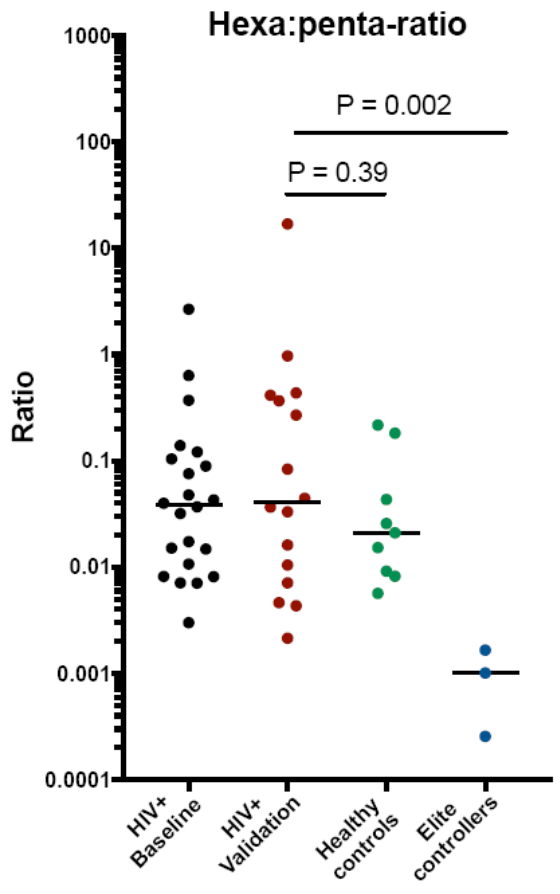

B

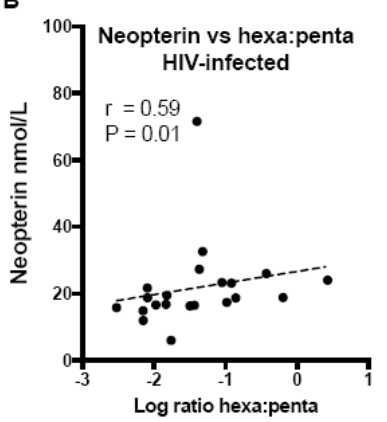

D

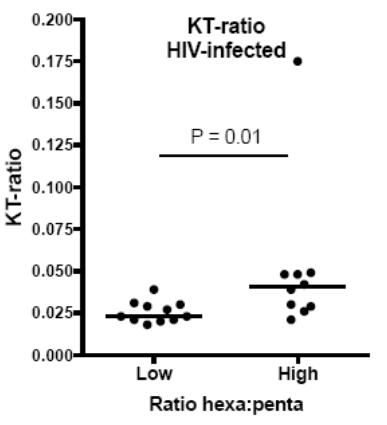

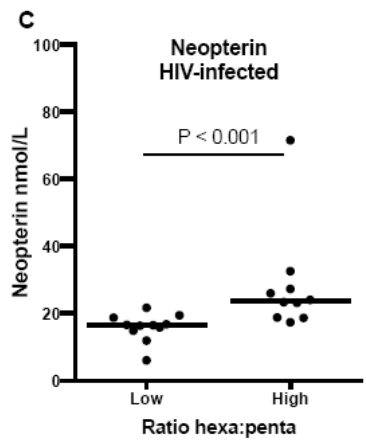

E

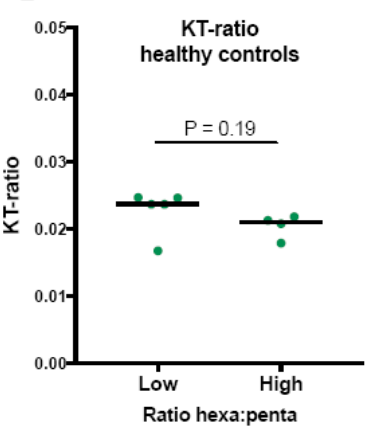


Figure 2. Effect of probiotic administration to HIV-infected individuals on type of LPS-

producing gut bacteria.

A A reduction in gut microbial genes related to LPS biosynthesis was seen in the probiotics group after 8 weeks of intervention. B The change in the predicted metagenome of genes related to LPS biosynthesis correlated to the change in relative abundance of gram-negative bacteria. The relative abundance of gut bacteria producing penta-acylated (C) and hexa-acylated LPS (D) before and after the probiotics intervention. E The ratio of hexa:penta-acylated LPS producers within the gut metagenome before and after the probiotics intervention.

Wilcoxon matched pairs test (a, c-e), Spearman's rho test (b).
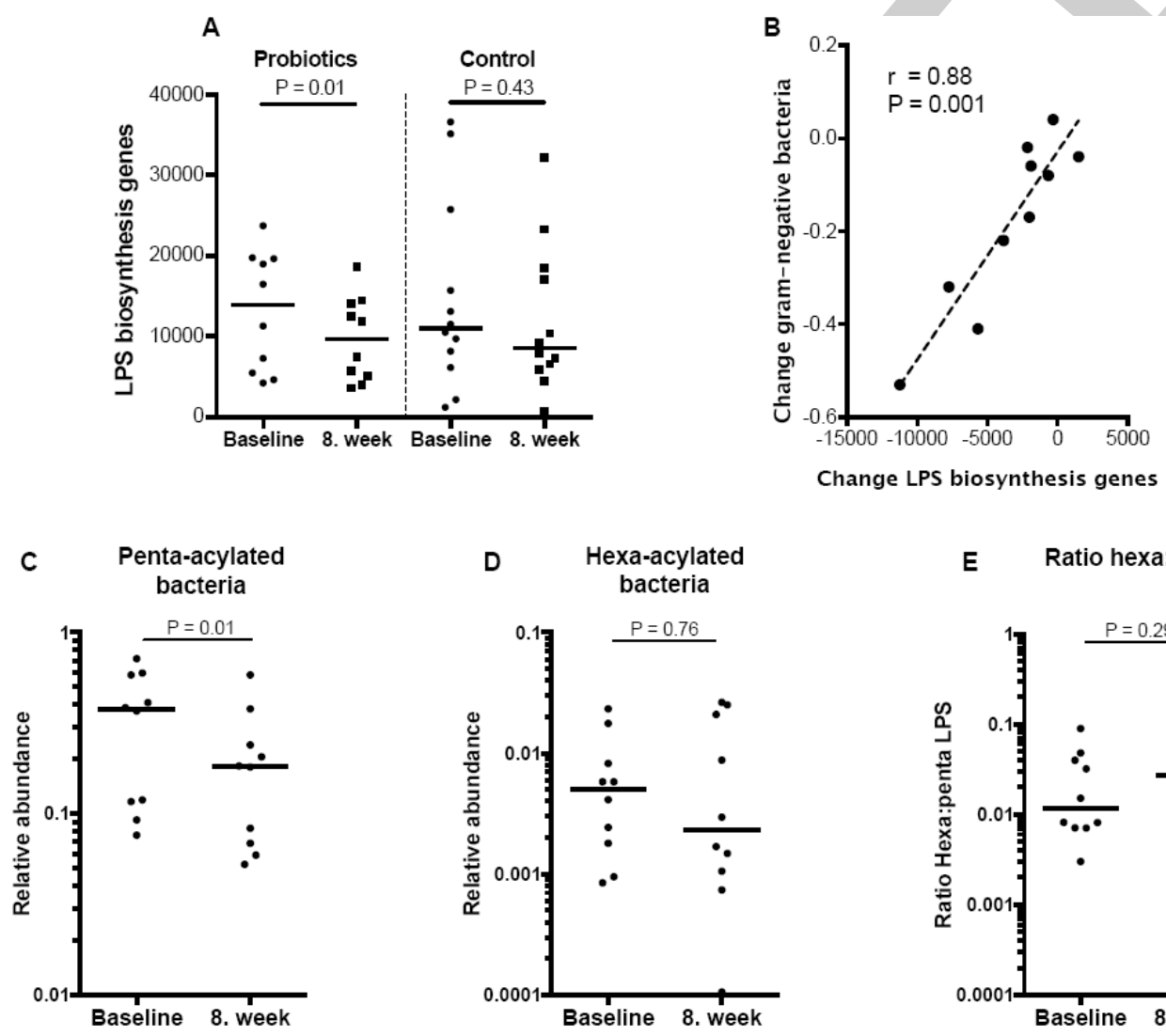

E Ratio hexa:penta

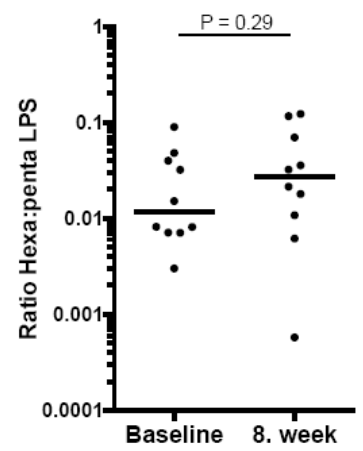


Figure 3. Correlations between gram-negative gut bacteria and bacteria producing pentaacylated LPS and plasma LPS levels

The change in the relative abundance of gram-negative gut bacteria correlated to the change in total plasma levels of LPS in the probiotics group (A). Likewise, the change in the relative abundance of bacteria producing non-inflammatory penta-acylated LPS correlated to changes in total plasma levels of LPS in the probiotics group (B). Spearman's rho test.

A

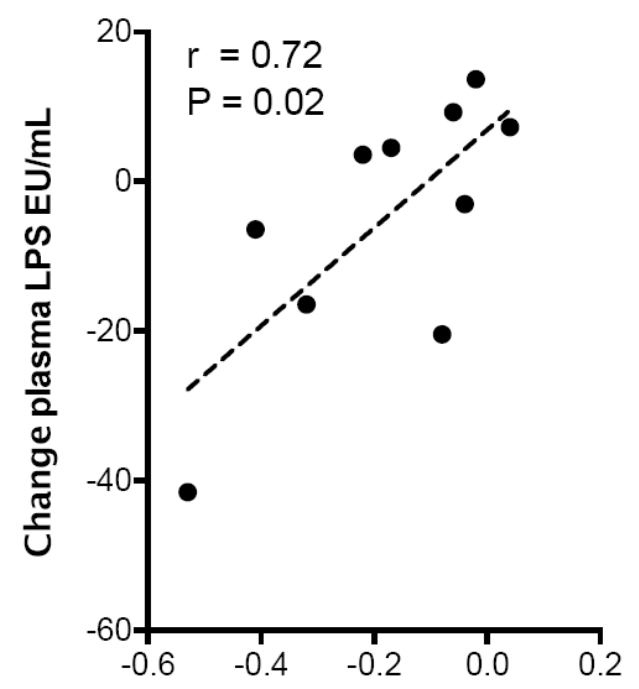

Change in relative abundance of gram-negative bacteria
B

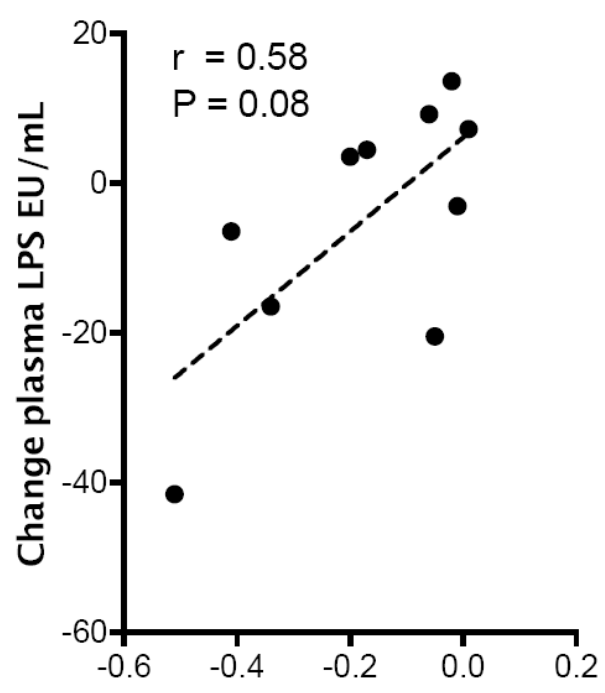

Change in relative abundance of bacteria producing penta-acylated LPS 
|Table 1 Characteristics of soluble markers in HIV-infected individuals in the probiotic intervention cohort.

Probiotics group $(\mathbf{n}=10)$

$\begin{array}{lccc} & \text { Baseline } & \text { 8. week } & \text { P-value } \\ \text { sCD14 }(\mu \mathrm{g} / \mathrm{ml}) & 1691 & 1601 & >0.10 \\ & (1320-2124) & (1302-2074) & \\ \text { LPS }(\mathrm{EU} / \mathrm{mL}) & 83 & 82 & >0.10 \\ & (81-95) & (74-97) & \\ \text { IL-6 }(\mathrm{pg} / \mathrm{mL}) & 1.2 & 1 & 0.09 \\ & (0.9-2.4) & (0.9-1.3) & \\ \text { D-dimer }(\mathrm{ng} / \mathrm{mL}) & 341 & 239 & 0.06 \\ & (230-505) & (149-417) & \\ \text { Neopterin }(\mathrm{nmol} / \mathrm{L}) & 18.7 & 19.2 & >0.10 \\ & (15.3-22.5) & (16.1-22.4) & \\ \text { KT-ratio } & 0.03 & 0.02 & >0.10 \\ & (0.02-0.03) & (0.02-0.04) & \\ \text { CRP }(\mathrm{mg} / \mathrm{L}) & 1.9 & 0.8 & \mathbf{0 . 0 5} \\ & (0.9-5) & (0.6-5.2) & \\ \text { I-FABP }(\mathrm{ng} / \mathrm{mL}) & 1231 & 1840 & >0.10 \\ & (925-1817) & (817-2188) & \end{array}$

Control group $(\mathbf{n}=12)$

Baseline 8. week P-value

$1692 \quad 1687 \quad>0.10$

$(1423-1928) \quad(1348-1978)$

$\begin{array}{ccc}93 & 95 & >0.10\end{array}$

$\begin{array}{ccc}1.4 & 1.4 & >0.10 \\ (0.9-3) & (1-1.8)\end{array}$

$\begin{array}{ccc}293 & 285 & >0.10 \\ (200-329) & (184-369)\end{array}$

$\begin{array}{ccc}18.8 & 19.1 & >0.10\end{array}$

$\begin{array}{ccc}(16.6-25.5) & (16.2-23.1) & \\ 0.03 & 0.03 & >0.10\end{array}$

$\begin{array}{ccc}0.03 & 0.03 & =0.10 \\ (0.02-0.05) & (0.02-0.04)\end{array}$

$1.2(1-5.7) \quad 2.1 \quad=0.10$

$\begin{array}{ccc}1247 & 1137 \\ (814-2322) & (855-2110)\end{array} \quad>0.10$
Between groups

\begin{tabular}{|c|c|}
\hline Baseline, $\mathrm{P}=$ & 8. week, $\mathrm{P}=$ \\
\hline$>0.10$ & $>0.10$ \\
\hline$>0.10$ & $>0.10$ \\
\hline$>0.10$ & $>0.10$ \\
\hline$>0.10$ & $>0.10$ \\
\hline$>0.10$ & $>0.10$ \\
\hline$>0.10$ & $>0.10$ \\
\hline$>0.10$ & $>0.10$ \\
\hline$>0.10$ & $>0.10$ \\
\hline
\end{tabular}

KT-ratio: kynurenine-tryptophan-ratio, a measure of tryptophan degradation.

Data are presented as median [interquartile range (IQR)] values. Wilcoxon matched pairs test in longitudinal analysis, Mann-Whitney U test between groups, not corrected for multiple testing. 
Table 2 Relative abundance of bacterial taxa in the gut of HIV-infected individuals.

\begin{tabular}{|c|c|c|c|c|c|c|c|c|}
\hline & \multicolumn{3}{|c|}{ Probiotics group $(n=10)$} & \multicolumn{3}{|c|}{ Control group ( $n=12)$} & \multicolumn{2}{|c|}{ Between groups } \\
\hline & Baseline & 8. week & P-value & Baseline & 8. week & P-value & Baseline, $\mathrm{P}=$ & 8. week, $P=$ \\
\hline Gram-negative & $\begin{array}{c}0.27 \\
(0.11-0.55)\end{array}$ & $\begin{array}{c}0.12 \\
(0.05-0.18)\end{array}$ & 0.01 & $\begin{array}{c}0.22 \\
(0.11-0.34)\end{array}$ & $\begin{array}{c}0.24 \\
(0.08-0.35)\end{array}$ & 0.70 & 0.63 & 0.31 \\
\hline Penta-acylated & $\begin{array}{c}0.38 \\
(0.11-0.59)\end{array}$ & $\begin{array}{c}0.18 \\
(0.07-0.27)\end{array}$ & 0.01 & $\begin{array}{c}0.21 \\
(0.1-0.37)\end{array}$ & $\begin{array}{c}0.24 \\
(0.1-0.35)\end{array}$ & 0.39 & 0.46 & 0.54 \\
\hline Hexa-acylated & $\begin{array}{c}0.005 \\
(0.002-0.01)\end{array}$ & $\begin{array}{c}0.002 \\
(\ll 0.001-0.02)\end{array}$ & 0.76 & $\begin{array}{c}0.02 \\
(0.01-0.07)\end{array}$ & $\begin{array}{c}0.007 \\
(0.002-0.032)\end{array}$ & 0.05 & 0.07 & 0.28 \\
\hline Bifidobacteriwn & $\begin{array}{c}0.01 \\
(0.001-0.02)\end{array}$ & $\begin{array}{c}0.02 \\
(0.005-0.06)\end{array}$ & 0.04 & $\begin{array}{c}0.02 \\
(0-0.04)\end{array}$ & $\begin{array}{c}0.012 \\
(0.003-0.021)\end{array}$ & 0.14 & 0.35 & 0.74 \\
\hline Lactobacillus & $\begin{array}{c}0 \\
(0-0.005)\end{array}$ & $\begin{array}{c}<0.001 \\
(<0.001-0.002)\end{array}$ & 0.06 & $\begin{array}{c}=0.001 \\
<0.001-0.003)\end{array}$ & $\begin{array}{c}<0.001 \\
: 00.001-0.001)\end{array}$ & 0.18 & 0.71 & 0.02 \\
\hline Bacteroides & $\begin{array}{c}0.19 \\
(0.04-0.39)\end{array}$ & $\begin{array}{c}0.03 \\
(0.002-0.09)\end{array}$ & 0.01 & $\begin{array}{c}0.04 \\
(0.01-0.08)\end{array}$ & $\begin{array}{c}0.07 \\
(0.01-0.1)\end{array}$ & 0.69 & 0.09 & 0.54 \\
\hline Parabacteroides & $\begin{array}{c}0.03 \\
(0.006-0.07)\end{array}$ & $\begin{array}{c}0.003 \\
(0.002-0.02)\end{array}$ & 0.01 & $\begin{array}{c}0.01 \\
(0-0.01)\end{array}$ & $\begin{array}{c}0.005 \\
(0.002-0.02)\end{array}$ & 0.31 & 0.01 & 0.88 \\
\hline Gram-positive & $\begin{array}{c}0.70 \\
(0.42-0.86)\end{array}$ & $\begin{array}{c}0.81 \\
(0.80-0.93)\end{array}$ & 0.01 & $\begin{array}{c}0.78 \\
(0.66-0.89)\end{array}$ & $\begin{array}{c}0.75 \\
(0.65-0.90)\end{array}$ & 0.70 & 0.28 & 0.42 \\
\hline
\end{tabular}

Data are presented as median [interquartile range (IQR)] values. Wilcoxon matched pairs test in longitudinal analysis, Mann-Whitney U test between groups,

not corrected for multiple testing. 